\title{
Energy-latitude dispersion patterns near the isotropy boundaries of energetic protons
}

\author{
V. A. Sergeev ${ }^{1}$, S. A. Chernyaeva ${ }^{1}$, S. V. Apatenkov ${ }^{1}$, N. Y. Ganushkina ${ }^{2,3}$, and S. V. Dubyagin ${ }^{3}$ \\ ${ }^{1}$ St. Petersburg State University, Ulyanovskaya 1, 198504 St. Petersburg, Russia \\ ${ }^{2}$ Department of Atmospheric, Oceanic and Space Sciences, University of Michigan, Ann Arbor, MI, USA \\ ${ }^{3}$ Earth Observation, Finnish Meteorological Institute, Helsinki, Finland \\ Correspondence to: V. A. Sergeev (victor@geo.phys.spbu.ru)
}

Received: 19 May 2015 - Revised: 23 July 2015 - Accepted: 30 July 2015 - Published: 31 August 2015

\begin{abstract}
Non-adiabatic motion of plasma sheet protons causes pitch-angle scattering and isotropic precipitation to the ionosphere, which forms the proton auroral oval. This mechanism related to current sheet scattering (CSS) provides a specific energy-latitude dispersion pattern near the equatorward boundary of proton isotropic precipitation (isotropy boundary, IB), with precipitation sharply decreasing at higher (lower) latitude for protons with lower (higher) energy. However, this boundary maps to the inner magnetosphere, where wave-induced scattering may provide different dispersion patterns as recently demonstrated by Liang et al. (2014). Motivated by the potential usage of the IBs for the magnetotail monitoring as well as by the need to better understand the mechanisms forming the proton IB, we investigate statistically the details of particle flux patterns near the proton IB using NOAA-POES polar spacecraft observations made during September 2009. By comparing precipitatedto-trapped flux ratio $\left(J_{0} / J_{90}\right)$ at $>30$ and $>80 \mathrm{keV}$ proton energies, we found a relatively small number of simple CSStype dispersion events (only $31 \%$ ). The clear reversed (waveinduced) dispersion patterns were very rare $(5 \%)$. The most frequent pattern had nearly coinciding IBs at two energies $(63 \%)$. The structured precipitation with multiple IBs was very frequent $(60 \%)$, that is, with two or more significant $J_{0} / J_{90}$ dropouts. The average latitudinal width of multiple IB structures was about $1^{\circ}$. Investigation of dozens of paired auroral zone crossings of POES satellites showed that the IB pattern is stable on a timescale of less than 2 min (a few proton bounce periods) but can evolve on a longer (several minutes) scale, suggesting temporal changes in some mesoscale structures in the equatorial magnetosphere.
\end{abstract}

We discuss the possible role of CSS-related and waveinduced mechanisms and their possible coupling to interpret the emerging complicated patterns of proton isotropy boundaries.

Keywords. Magnetospheric physics (energetic particles precipitating)

\section{Introduction}

In the loss-cone trap geometry of the geomagnetic field, the adiabatic motion of charged particles conserves the empty loss cone in the particle distributions. However, in the regions where the particle gyroradius $\rho$ is comparable to the magnetic curvature radius $\left(R_{c}\right)$ and adiabatic approximation is violated (when, roughly, $R_{c} / \rho \leq 10$; Alfven and Falthammar, 1963), pitch-angle scattering occurs, which fills the loss cone providing the particle precipitation into the ionosphere. In the Earth's magnetosphere, for the protons with energies of a few $\mathrm{keV}$ to hundreds of $\mathrm{keV}$, the boundary between adiabatic and non-adiabatic particle motion is located near the center of the tail current sheet on the nightside at $r \sim 6-9 R_{\mathrm{e}}$ (e.g., Sergeev and Tsyganenko, 1982; Yue et al., 2014). This mechanism (later referred to as the current sheet scattering, or CSS-related) is rather well investigated: it is now widely recognized as the main mechanism which causes the nearly isotropic proton angular distributions in the tail plasma sheet (e.g., Ganushkina et al., 2005; Wang et al., 2012; Yue et al., 2014) and which provides the extended proton precipitation into the auroral oval (Donovan et al., 2003; Meurant et al., 2007). In the ionosphere, the distinctive signature of the CSS mechanism is the energy-dispersed equatorward 
boundary of the isotropic precipitation (isotropy boundary, IB), whereas the poleward boundary is usually the boundary where the particle flux becomes very low, e.g., at the polar cap boundary or radiation belt termination. In the tail current sheet, the normal $\left(B_{z}\right)$ and radial $\left(B_{r}\right)$ magnetic field components control the angular scattering amplitude. The criterion separating full loss-cone coverage (strong scattering) from partial (incomplete) cone-filling conditions is approximately $K=R_{c} / \rho \approx B_{z}^{2} /\left(\mathrm{d} B_{r} / \mathrm{d} z \cdot G\right) \cong 8$, where $G=\mathrm{mV} / e$ is the particle rigidity (e.g., Sergeev and Tsyganenko, 1982; Delcourt et al., 1996). With increasing tail current (that is, with $B_{z}$ decreasing in the inner magnetosphere), the location of the $K \sim 8$ boundary shifts earthward and the latitude of its ionospheric projection (isotropy boundary, IB) shifts equatorward too, by roughly $1.5^{\circ}$ of corrected geomagnetic latitude (CGLat) per each $10 \mathrm{nT} B_{z}$ depression at geostationary orbit. High correlation systematically exists between the observed $B_{z}$, or field inclination at the geostationary orbit (GEO), and the latitude of proton IB (Sergeev et al., 1983, 1993; Newell et al., 1998) or its optical proxies (Donovan et al., 2003; Meurant et al., 2007). Moreover, statistically the IB locations predicted using different data-based magnetospheric models usually agree with their observed locations within the uncertainty of those statistical models (e.g., Sergeev and Gvozdevsky 1995; Shevchenko et al., 2010; Yue et al., 2014), which is evaluated to be a couple degrees of latitude (e.g., Shevchenko et al., 2010; Nishimura et al., 2011).

In the magnetotail configuration with $B_{z}$ monotonically decreasing outward, the CSS mechanism provides a sharp cutoff and a specific rigidity-latitude dispersion pattern, with the isotropy boundary being at a higher (lower) latitude for protons with lower (higher) energy (as well as for much higher latitudes for electrons). It can be used to confirm the CSS origin, if observed. Such a dispersion pattern was documented in magnetospheric observations (West et al., 1978), first observed for energetic electrons and protons by Imhof et al. (1977) near the ionosphere, and later also confirmed on many occasions by NOAA-POES observations of $>30 \mathrm{keV}$ particles (see, e.g., Sergeev et al., 2012, and references therein) and by FAST observations of $<20 \mathrm{keV}$ protons (Yue et al., 2014). However, recently, Liang et al. (2014) also reported a comparable number of FAST auroral zone crossings showing the reverse type of dispersion of $1-20 \mathrm{keV}$ protons. They also provided wave observations in the magnetically conjugate equatorial magnetosphere region as well as theoretical arguments supporting the idea that such reverse dispersion precipitation was probably produced by the wave-particle interaction with the electromagnetic ion cyclotron (EMIC) waves. These results call into question the role and applicability of the CSS mechanism. They also suggest a number of questions to answer; for example, are these results specific for the low-energy part of the proton spectrum? What is the morphology of the proton precipitation in the energy range from tens to hundreds of keVs near proton IB? How are these results compatible to the bulk of evidence supporting the CSS-related pitch-angle scattering of plasma sheet protons?

In fact, evidence of wave-particle interaction is also ample in the literature, especially for electrons (e.g., Horne et al., 2005). For energetic protons, which are the exclusive topic of interest in this paper, two types of nightside precipitation were previously identified. One is several degrees latitudewide long-duration and azimuthally extended precipitation with a partly filled loss cone $\left(J_{0} / J_{90}<1\right)$, which is observed equatorward from the proton isotropy boundary, called the low-latitude proton precipitation (LLPP) (Gvozdevsky et al., 1997). The LLPP particle flux significantly increases during intense substorms and then decays with a long characteristic timescale of $\sim 9 \mathrm{~h}$. Because of that it may also be found during very quiet time periods following the substorms. The long decay and the observed loss-cone anisotropy imply a moderate pitch-angle diffusion of energetic protons. During substorm, the $J_{0} / J_{90}$ flux ratio increases up to $\sim 1$, so it may obscure the true isotropy boundary formed by the CSS mechanism, especially during storm times and in the evening sector (Dubyagin et al., 2013). Another well-documented precipitation type is the intense detached proton precipitation regions (often appearing as spikes) on the IB equatorward side (Yahnin and Yahnina, 2007; Usanova et al., 2010). These localized precipitations are known to correlate with isolated hydrogen auroral emission patches, and they show a close relationship to the Pc1-range pulsations, which is evidence for the interaction with EMIC waves. On many occasions these latitudinally detached wave-induced precipitations are observed in vicinity of the plasmapause. It is not known for sure whether similar wave-induced precipitation can be observed in the more poleward region near the proton isotropy boundary.

Motivated by the potential importance of the proton IBs as tracers of the magnetotail configuration as well as by the need to better understand the mechanisms forming the proton IBs, in this paper, we investigate the patterns of trapped $\left(J_{\mathrm{t}}\right)$ and precipitated $\left(J_{\mathrm{p}}\right)$ proton fluxes at a few energies near the proton IB using observations made by NOAA-POES polar spacecraft. We select September 2009 as the period for this statistical study for two reasons. First, we expected that possible wave effects could be minimized during weak magnetospheric activity at the solar minimum. Second, during this month, two POES spacecraft (NOAA-18 and NOAA19) flew in the same orbital plane but at slightly different altitudes and with slightly different orbital periods, providing an opportunity to investigate how stable the IB patterns are (which had never been done before) and which kind of evolution they display.

\section{Observations}

We use the observations of trapped $\left(J_{\mathrm{t}}\right)$ and precipitating $\left(J_{\mathrm{p}}\right)$ energetic ion and electron fluxes provided by the Space 
Environment Monitor-2 (SEM-2)/Medium Energy Proton and Electron Detector (MEPED) and Total Energy Detector (TED) instruments onboard the low-altitude $(\sim 850 \mathrm{~km})$ polar-orbiting NOAA-POES spacecraft. Data are available at $2 \mathrm{~s}$ time resolution from http://ngdc.noaa.gov/stp/satellite/ poes/. Here, we use the low-energy MEPED channels for protons, nominally $30-80 \mathrm{keV}(\mathrm{P} 1), 80-240 \mathrm{keV}(\mathrm{P} 2)$ and 240-800 keV (P3) (Evans and Greer, 2004); the latter channel provides a low count rate during quiet periods and is not used in the following statistical study of energy dispersion. For survey purposes, we also use the total energy flux of precipitated auroral electrons $\left(\mathrm{J} E_{\mathrm{e}}\right)$ and auroral protons $\left(\mathrm{J} E_{\mathrm{p}}\right)$ from the TED instrument (covering $0.05-20 \mathrm{keV}$ energies). Sharp equatorward cutoff of the TED proton energy flux provides a proxy of proton isotropy boundary (IB) at $10-20 \mathrm{keV}$ energies, known as the b2i boundary (Newell et al., 1998).

\subsection{Particle flux structure near the isotropy boundaries and its stability: analysis of "string-of-pearls" oval-skimming passes of the NOAA-POES spacecraft pair}

A variable particle flux structure observed near the isotropy boundaries poses questions which need to be answered: how stable are these structures/dispersion patterns, and do they evolve in time and longitude (and at which scales)? To partly answer these questions, we analyze the conjunction orbits of the NOAA-19 and NOAA-18 satellites. NOAA-19 was launched in February 2009 into a Sun-synchronous orbit with the same orbital plane as NOAA-18 had. Due to the difference in their orbital periods by the mid-September 2009, the spacecraft achieved a flying configuration in which they crossed the auroral zone nearly simultaneously (within $1 \mathrm{~min}$ ) at nearly the same latitude and longitude. Also, at the beginning (end) of September, the NOAA-19 satellite led (trailed) the NOAA-18 satellite by roughly $5 \mathrm{~min}$, allowing for the temporal stability of the IB structure to be looked into. Such a "string-of-pearls-type" flying configuration is helpful to resolve the temporal/spatial origin of the observed IB structures. From all such observations in September 2009, we further selected the oval-skimming crossings of the southern auroral zone, during which two spacecraft moved along the same orbit. This orbit crossed the isotropy boundaries on the nightside from low latitude, then passed along the auroral oval, and then crossed again the isotropy boundaries toward lower latitudes on the duskside.

Figure 1 provides an example of quiet-time oval-skimming crossing by two spacecraft on 13 September 2009. During the nightside crossings (left panels), both spacecraft observe a kind of standard dispersion with a low-energy $(<20 \mathrm{keV})$ flux peak boundary (b2i) staying at higher latitude $\left(65.5^{\circ}\right)$ compared to the major precipitated flux decrease of 30 and $80 \mathrm{keV}$ protons, which cluster near $\sim 65^{\circ}$ CGLat. It also shows some dropouts of precipitated flux, later referred to as the IB structure. Obviously, the precipitated flux structure in this case is more complicated than the classical sharp dropout of the $J_{0} / J_{90}$ ratio expected at the IB. Different from that, both spacecraft in the duskside crossing (right panels) show virtually no dispersion, and the proton isotropy boundaries cluster near the same location $\left(66.7^{\circ}\right)$ in the 30 and $80 \mathrm{keV}$ channels where the b2i boundary is also observed. Such an IB grouping in the nearby energy channels is rather frequently observed, as will be shown below. General features of each crossing are reproduced at both spacecraft, which flew one after the other with a time delay of $1.5 \mathrm{~min}$ during this event. Note that the bounce period of $100 \mathrm{keV}$ protons at $\mathrm{L} \sim 8$ is about half a minute, so the flux pattern looks stable on the scale of few bounce periods, and therefore it represents a truly spatial pattern.

Figure 2 provides two examples of a more complicated and dynamical situation in which between two crossings the precipitated fluxes evolve considerably in the localized latitudinal range near the IBs. Note that the trapped fluxes (black curves) as well as the precipitated fluxes in two regions located poleward and equatorward of this latitudinally localized $\sim 1^{\circ}$ CGLat-wide region change little between the two crossings. In the first case (a), during first passage, NOAA19 observed a sharp isotropy boundary in the $30 \mathrm{keV}$ channel at $-66.5^{\circ}$, whereas 4 min later the clear IB in this channel was recorded at $65.5^{\circ}$ by NOAA-18. Similarly, in the second case (b), after the IB recorded by NOAA- 19 at $67.5^{\circ}, 4 \mathrm{~min}$ later NOAA-18 observed a large drop in precipitated flux between 67.5 and $68.5^{\circ}$. The changes in the $80 \mathrm{keV}$ energy channel are similar but smaller in magnitude. In these examples, considerable $\left(\sim 1^{\circ}\right.$ CGLat-wide) latitudinal IB shifts do not occur because of global flux redistribution or because of the overall shift of the entire pattern. Rather, they occur due to the localized enhancement or depletion of the precipitated flux in the localized region near the previous IB (without obvious changes in the surrounding regions and without any large change in the trapped flux distribution). As we shall argue later, such a kind of evolution can be characteristic of the resonant wave-particle interaction suddenly enhanced in this localized region. We should also note that, in the $80 \mathrm{keV}$ channel, the transition of $J_{0} / J_{90} \sim 1$ to the small values in first case occurred very gradually, similar to what is expected (and typically observed) near the boundary between adiabatic and non-adiabatic particle scattering in the magnetotail.

Visual inspection of 27 oval-skimming crossings allowed us to quantitatively characterize the stability of the observed flux structure, classified into three categories (1: no IB dispersion; 2: standard IB dispersion; and 3: structure near the IB), depending on the time delays, dT, between the passages of two spacecraft. Small latitudinal shifts of the entire pattern were ignored. By comparing the recordings of two spacecraft, the IB flux pattern was found to be the same in seven of nine cases if $\mathrm{dT}<2 \mathrm{~min}$, in five of nine cases if dT was 2 to $4 \mathrm{~min}$, and in three of nine cases for $\mathrm{dT}=4-6 \mathrm{~min}$. This limited statistical study implies that the flux structure near the proton isotropy boundary is conserved on a few bounce 

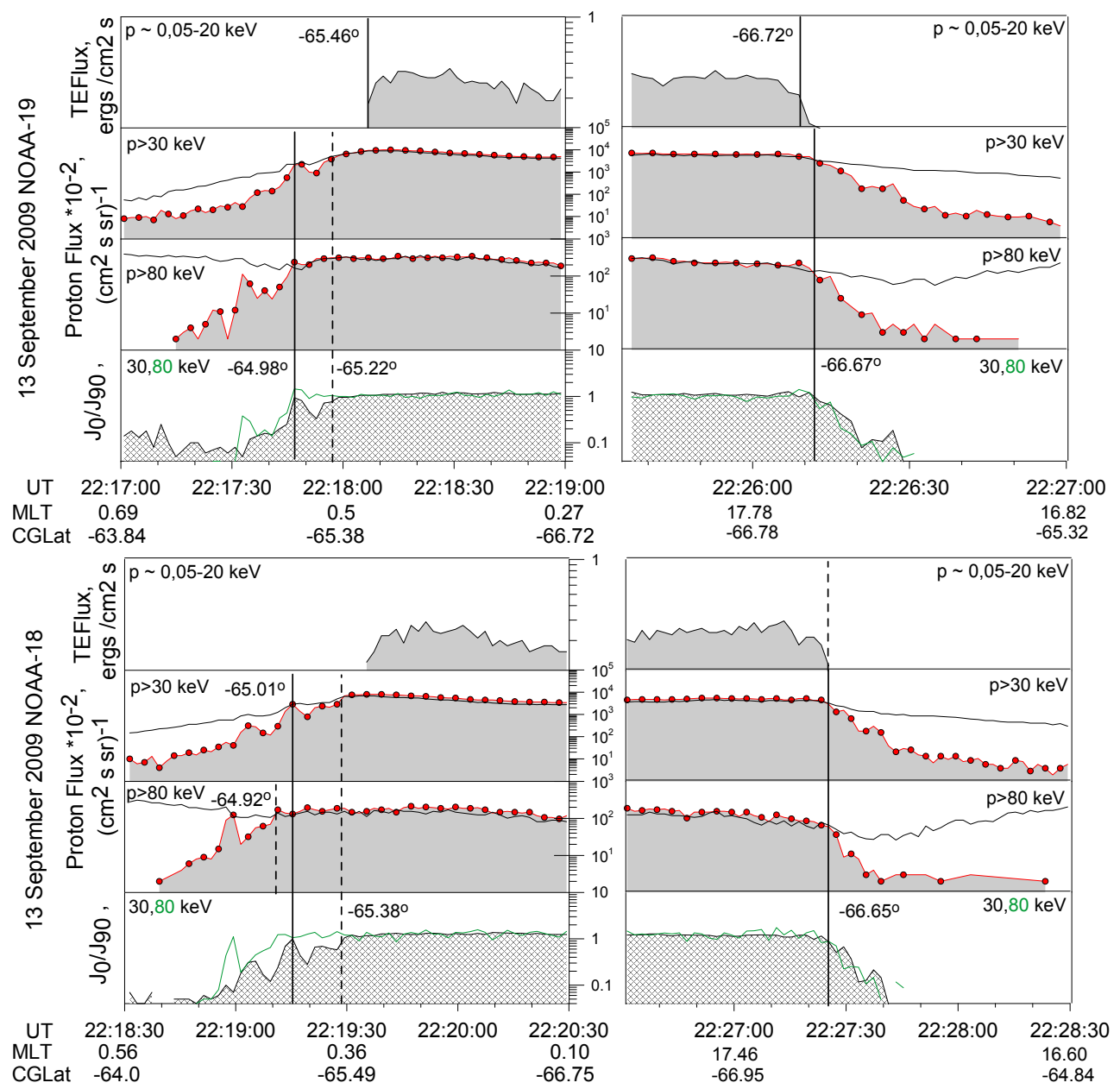

Figure 1. Proton flux variations during oval-skimming passages by the NOAA-19 (top) and NOAA-18 spacecraft (bottom). Spaces below the precipitated flux $\left(J_{0}\right)$ are shaded grey, and trapped flux is shown by the solid line. Dots show the measurements at $2 \mathrm{~s}$ resolution. The bottom panel for each spacecraft shows the precipitated-to-trapped flux ratio for two energy channels ( $30 \mathrm{keV}$ - black; $80 \mathrm{keV}$ - green). Vertical lines mark the sharp flux ratio changes which can be used to identify the isotropy boundaries.

timescales, but this spatial structure can evolve significantly on the timescale of the order of $5 \mathrm{~min}$ or more during quiet periods.

\subsection{IB energy dispersion patterns and their occurrence and parameters}

To illustrate the dispersion pattern in the global context and its variability, Fig. 3 (left panel) examines the latitudinal locations of the isotropy boundaries at different energies in three dozen NOAA-19 passes, which occurred during a very quiet period on 26 September 2009 followed by a few episodes of intense substorm activity later on this day and on the following day. To exclude possible local time variations, only the postmidnight crossings (00:00-04:00 MLT) are plotted here. During both quiet and active times, on many occasions we observe the IB latitudes decreasing in the order of increasing particle rigidity (from left to right): $30 \mathrm{keV}$ elec- trons; then $<20,30$ and $80 \mathrm{keV}$ protons; and finally $250 \mathrm{keV}$ protons (during rare cases when their count rates exceeded 10 protons $\mathrm{cm}^{-2} \mathrm{~s}^{-1}$ ). This is the standard pattern predicted by the CSS mechanism. That pattern does not change much between quiet and active time periods, except for the latitudinal shifts of the entire pattern attributed to the changing stretching of the magnetotail field lines. In addition, it is not altered much in the presence of LLPP (right column of Fig. 3), indicating the enhanced wave activity in the inner magnetosphere. However, some apparent deviations from the simple behavior predicted by the CSS mechanism are evident in Fig. 3. Particularly, the reverse order of latitudinal dispersion (higher-energy IB stays at higher latitude compared to low-energy IB) is sometimes observed. As different from inverse dispersion patterns of low-energy protons shown by Liang et al. (2014) which occupied their entire broad energy range $1-20 \mathrm{keV}$, in the high-energy range such global anomalies are infrequent. As a rule, only one channel stays at an 

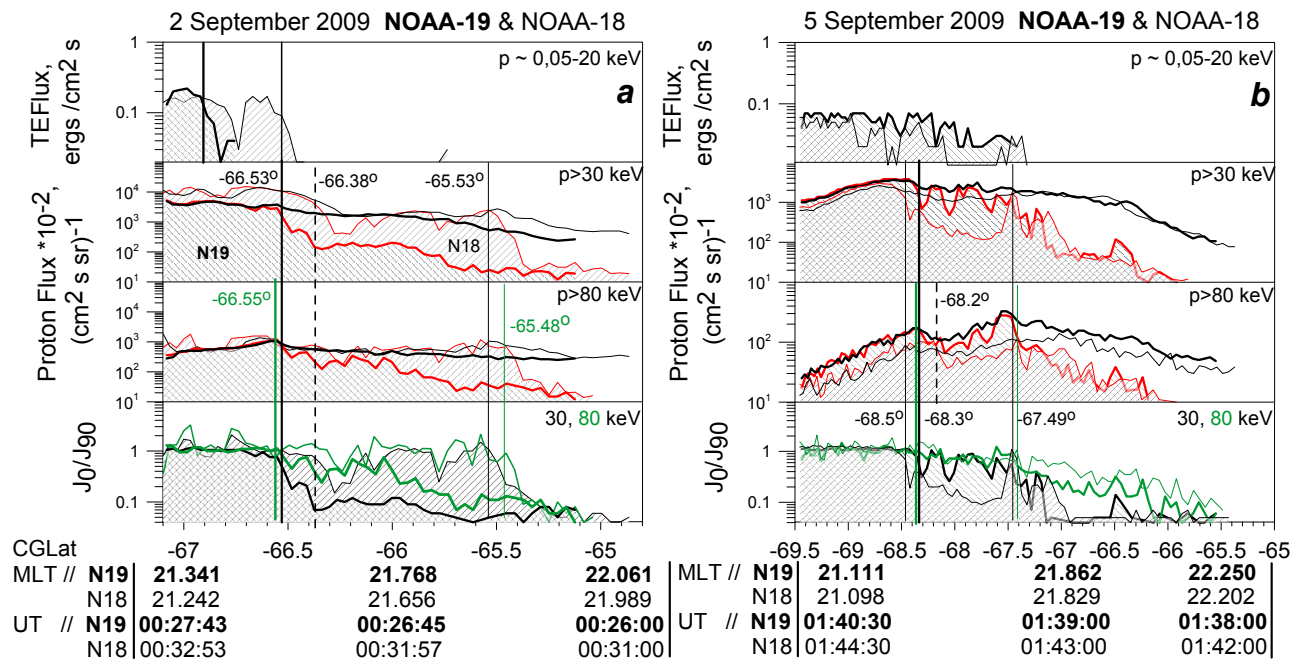

Figure 2. Examples of latitudinal profiles of time-evolving precipitation near the isotropy boundary observed by NOAA-19 (bold lines) and NOAA-18 (thin lines) observed on 2 September 2009 with a 5 min delay (a) and on 5 September (b) with an $\sim 4$ min time delay. Black and green color of the lines in the bottom panel as well as vertical lines correspond to the 30 and $80 \mathrm{keV}$ energy channels. Red lines show the precipitated flux.

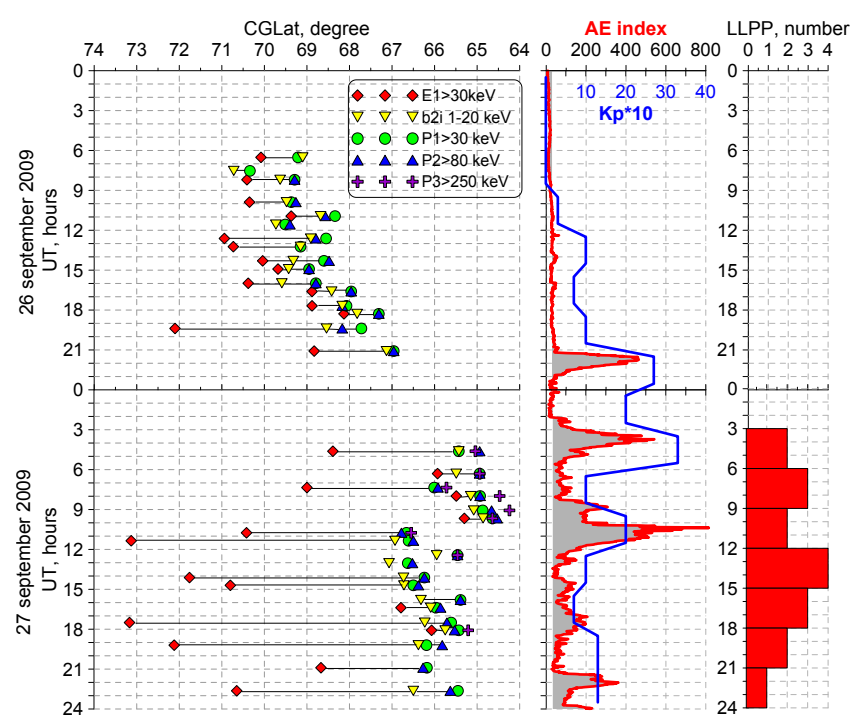

Figure 3. Survey of activity and IB dispersion patterns on 26 and 27 September 2009. From left to right: IB locations at different energies during NOAA-19 crossings in postmidnight MLT; AE and Kp activity; and number of LLPP precipitation events (equatorward of proton IB locations) in $3 \mathrm{~h}$ UT bins identified in the NOAA-19 data.

anomalous location, whereas locations of other channels are ordered as usual (see, for example, the crossing at 19:30 UT on 26 September, where $30 \mathrm{keV}$ IB occurs at the most equatorward location).

Another, more frequent deviation from the standard pattern occurs when two (rarely three) IBs group at the same latitude in the nearby channels. This can be seen at the last pass on 26 September (all boundaries coincided) and first pass on 27 September (b2i and $30 \mathrm{keV} ; 80$ and $250 \mathrm{keV}$ IBs are also grouped by pairs). With a $2 \mathrm{~s}$ sampling rate and $100 \mathrm{~min}$ orbital period of the NOAA-POES spacecraft, the latitudinal resolution of particle detectors is about $0.12^{\circ}$; therefore, as is shown below, we formally define two boundaries as separated in space if their IB latitudes are separated by $>0.2^{\circ}$ (that is, by more than two time resolution steps), whereas those recorded within two time steps are considered as being coincident in space. For a numerical estimate of CSS-predicted energy dispersion, we computed the predicted isotropy boundaries at the midnight meridian using the criterion $K_{\mathrm{cr}}=R_{c} / \rho=8$ for the T96 model (Sergeev et al., 1983). It was found that the latitudinal difference between 30 and $80 \mathrm{keV}$ proton IBs varies slightly for different solar wind and interplanetary magnetic field (IMF) conditions, and is about $0.6-0.7^{\circ}$ CGLat. Visually, this value is larger compared to the IB latitudinal difference observed in the majority of cases shown in Fig. 3, so this aspect requires a quantitative investigation.

To study the occurrence and distribution of dispersion patterns quantitatively, we can basically rely upon the observations in the two proton channels $>30 \mathrm{keV}$ (p1) and $>80 \mathrm{keV}$ (p2), which provide sufficient count rates for both quiet and disturbed conditions. We formally define the isotropy boundary as the start of significant (more than a factor of 3 ) and sharp (shorter than $10 \mathrm{~s}$ ) dropout of the $J_{0} / J_{90}$ flux ratio from its nearly isotropic state $\left(J_{0} / J_{90} \sim 1( \pm 10 \%)\right)$ on the equatorward side from the isotropic precipitation zone. In Figs. 4 and 5, we illustrate a few types of possible dispersion patterns. Besides precipitated and trapped flux variations, the bottom panel in these figures shows the $J_{0} / J_{90}$ ratios, cor- 
rected for secular variation in channel energies (according to Asikainen et al., 2012). Low-energy proton precipitated flux is also shown for reference.

The first example (Fig. 4a) illustrates a simple standard dispersion pattern of the type predicted by the CSS mechanism (our StandD group). The low-energy b2i boundary (total energy flux drop in the equatorward direction in the upper panel) and isotropy boundaries at 30 and $80 \mathrm{keV}$ stay at $70.5,70.2$ and $69.9^{\circ}$ CGLat, respectively, in the Southern Hemisphere. For this group of events (StandD group) we require $\mid$ IBL30 $|-|$ IBL80 $\mid>0.2^{\circ}$. Next, Fig. 4b illustrates the case with virtually no IB dispersion between the 30 and $80 \mathrm{keV}$ channels; here both IBs stay at $69.3^{\circ}$, and in this case the b2i boundary can be determined slightly poleward. Our formal criterion imposed for this group (NoD group) was $|\operatorname{IBL}(\mathrm{p} 1)-\operatorname{IBL}(\mathrm{p} 2)|<0.2^{\circ}$ ).

The behavior illustrated in Fig. $4 \mathrm{c}$ is unusual: here in the $30 \mathrm{keV}$ channel, the IB stays at a lower latitude than in the $80 \mathrm{keV}$ channel, which is inconsistent with the predictions of the CSS mechanism. This is representative of a group of anomalous dispersion (ANOM) events, for which we require $\mid$ IBL80 $|-|$ IBL30 $\mid>0.2^{\circ}$. In this case (but not necessarily always), the b2i boundary does not follow the anomaly and is slightly shifted to a higher latitude. We note that there were small variations in the $J_{0} / J_{90}$ flux ratio in the $\sim 1$ CGLatwide zone poleward of the IB which did not exceed a factor of 2. If these variations were stronger (exceeding a factor of 3 ), this event would be classified as part of the next group, the group of structured IB.

Figure 5a shows an example of two temporal drops of $J_{0} / J_{90}$ ratio (exceeding a factor of 2 ) in the $30 \mathrm{keV}$ channel before the final sharp drop (IB2) taken as a formal isotropy boundary, which in this case shows no IB energy dispersion between the 30 and $80 \mathrm{keV}$ channels. A second transient variation is also seen simultaneously in the $80 \mathrm{keV}$ channel, with the $J_{0} / J_{90}$ drop being larger in the $30 \mathrm{keV}$ than in the $80 \mathrm{keV}$ channel (green trace above the black trace in the bottom panel; this is what we expect from the CSS mechanism). These are quite typical features for this group of structural events (STR group), which otherwise includes a variation in the number of transient drops, in their latitudinal extension, in the dispersion at the IB2 boundary, etc. An important parameter for this group could be the latitudinal difference between the first and last $J_{0} / J_{90}$ drops (IB1-IB2, in this case about $1.1^{\circ}$ ), which shows the spread of possible locations of the IB. One more useful potential indicator of CSS-related isotropy boundary may also be the precipitated flux peak location (IB0).

Three other examples also include the pronounced (anisotropic) LLPP precipitation extending equatorward from the isotropy boundary. Formally, we identified the LLPP if steady $J_{0} / J_{90}>0.1$ in the 30 or $80 \mathrm{keV}$ channel (or both) exists in the region extending for more than $1.5^{\circ}$ CGLat equatorward of isotropic precipitation. In the case of Fig. 5b, the IB determination does not suffer from LLPP presence, whereas in other cases (Fig. 5c, d) its existence causes some uncertainty. This is due to significant LLPP flux variations which can be mixed with true IB (Fig. 5c), or due to a gradual decrease in $J_{0} / J_{90}$ with decreasing latitude, which makes the IB determination questionable (Fig. 5d). Therefore, when compiling statistics and studying the occurrence of various IB patterns, we have to carefully take into account the presence of LLPP and its possible interference with isotropic precipitation.

Surprisingly, although September 2009 was one of the most quiet months of the deepest solar cycle minimum, there are still a lot of wave-scattered LLPPs - they were observed in one-third of all passes. Figure 6 shows the daily occurrence of NOAA-19 nightside auroral crossings showing the LLPP signatures. Some days have a lot of LLPP samples, suggesting widespread enhanced wave activity in the magnetosphere, while some do not show LLPP at all. Similar to Gvozdevsky et al. (1997), we notice grouping of the LLPP days, with LLPPs starting together with enhanced AE and $\mathrm{KP}$, and continuing (subsiding) for 1-2 days after the activity diminished, as can also be noticed in Fig. 3. Different from Gvozdevsky et al. (1997), the activity magnitude required to produce the LLPP is rather modest, being only about 300$400 \mathrm{nT}$ in the $\mathrm{AE}$ index and $\mathrm{Kp} \sim 2-3$ in our case.

\subsection{Statistics of different isotropy boundary dispersion patterns}

To statistically characterize the occurrence and the activity and MLT dependencies of different IB patterns, we analyzed all passes of NOAA-19 in September 2009 (orbiting in postmidnight-postnoon orbital plane) and MetOp-2 (a POES-type spacecraft carrying the same instruments but flying in the premidnight-prenoon plane) observations during first two weeks of September 2009, altogether providing 558 isotropy boundary crossings on the nightside. The occurrence numbers of different IB patterns are summarized in Table 1 and illustrated in Figs. 7 and 8. The relative occurrence of different dispersion types, displayed in Fig. 7a, shows a rather weak MLT variation, except for a slight tendency towards appearance of anomalous dispersion events in the postmidnight sector. The number of anomalous events is, however, very small $(5 \%)$. The CSS-like (normal) dispersion is seen in one-third of all events. The most frequent "no-dispersion" events group (63\%) also does not display significant MLT variation (Fig. 7b). Similarly, Fig. 7c shows no significant MLT-dependent changes for appearance of the structured IBs, and the same is the case for appearance of widespread LLPP precipitation on the low-latitude side of the proton isotropy boundary. The structured IBs are very frequent (they are seen in $60 \%$ of all cases) and the LLPPs are observed in $41 \%$ of all events. In addition, Table 1 illustrates that the occurrence of different types and amounts of latitudinal dispersion does not depend on the presence of the LLPPs. 

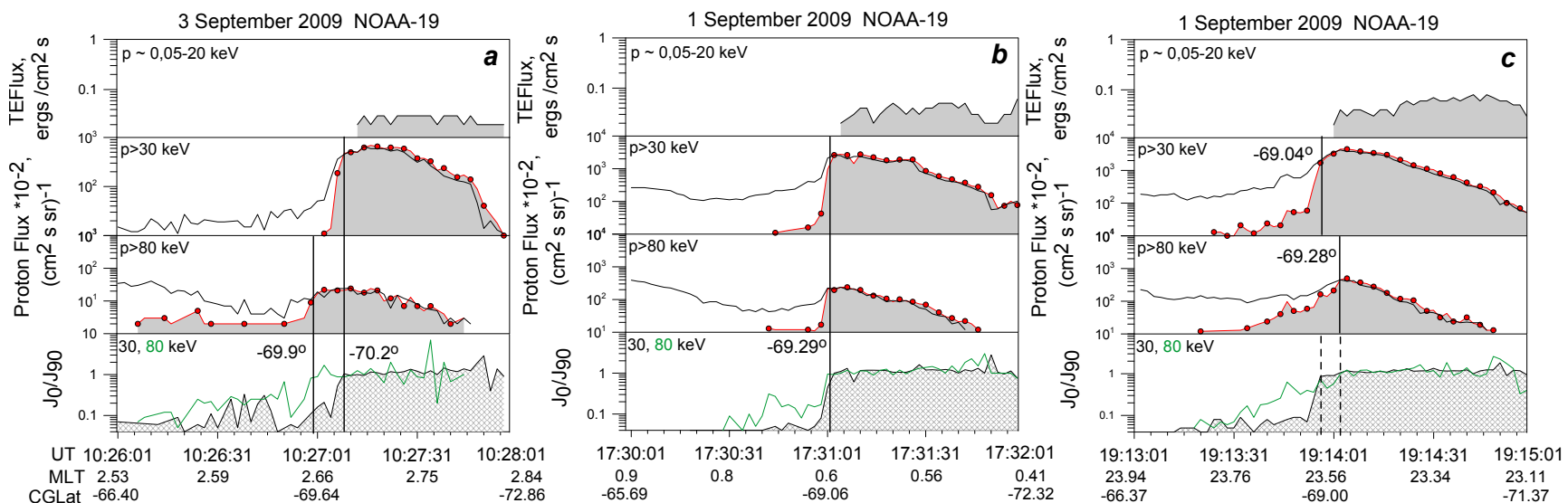

Figure 4. Examples of standard-type dispersion (a), no IB dispersion (b), and anomalous dispersion (c).
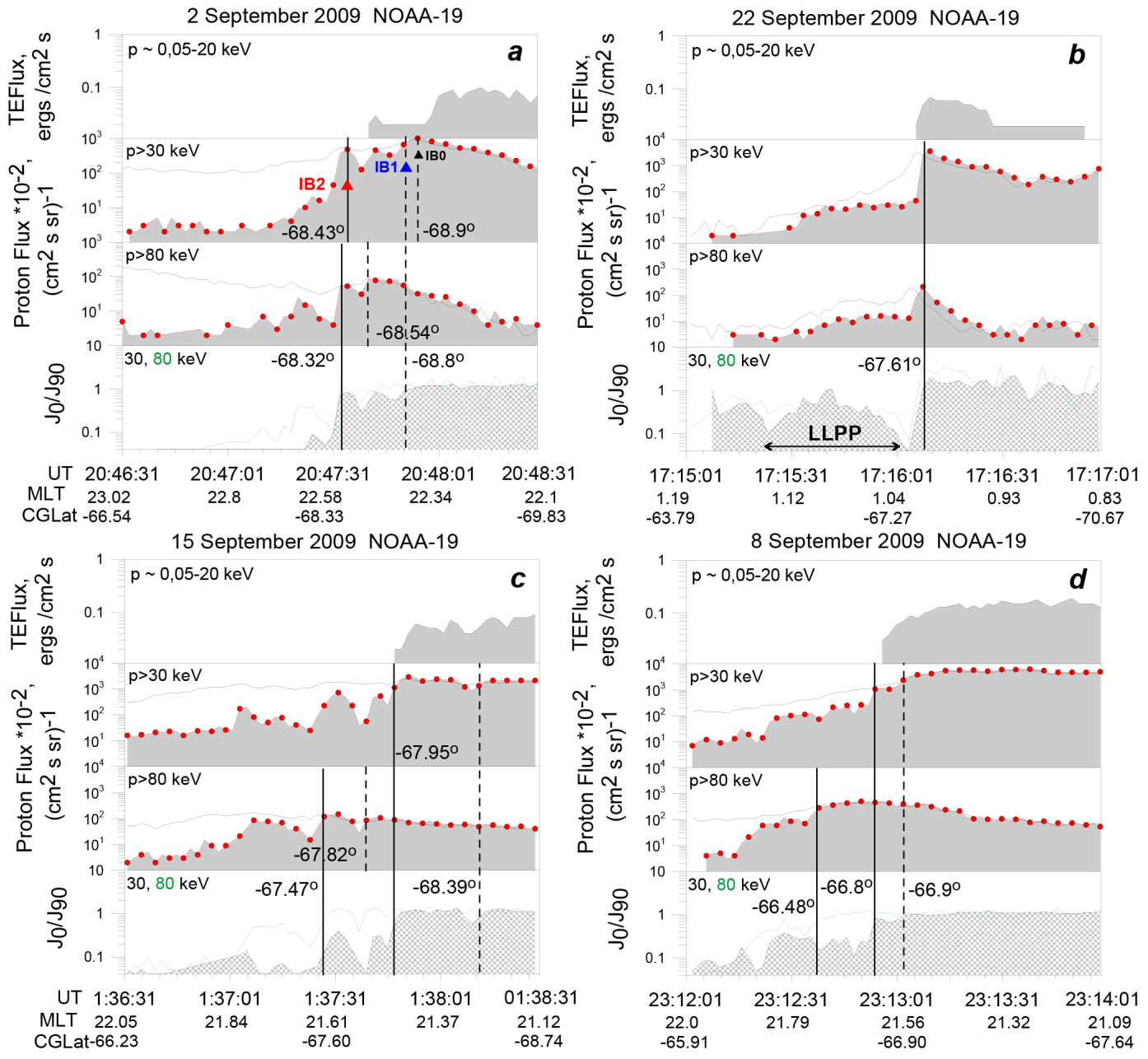

Figure 5. Examples of more complicated behavior including structured IB (with no IB dispersion) (a), no IB dispersion case with adjacent but separated LLPP (b), well-defined structured IB at the LLPP background (c), and "gradually developing IB" that interfered with LLPP background (d). The structured IBs have been characterized by the peak precipitated flux (IB0), poleward-most $J_{0} / J_{90}$ dropout (IB1) and equatorward-most $J_{0} / J_{90}$ dropout (IB2), used also as the IB proxy in the statistical studies. 


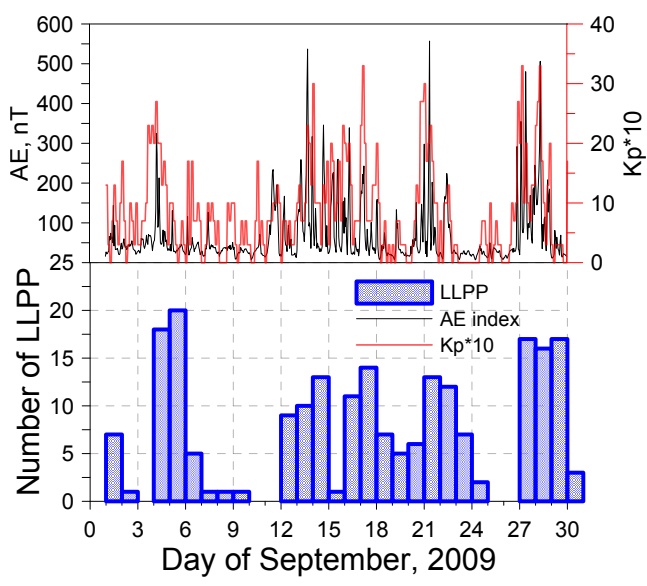

Figure 6. Magnetic activity and LLPP daily occurrence in September 2009 according to NOAA-19 observations.

Table 1. Occurrence of latitudinal shifts of the $30 \mathrm{keV}$ and $80 \mathrm{keV}$ proton IBs (and average IB latitudinal dispersion)

\begin{tabular}{llll}
\hline $\begin{array}{l}\text { Type } \\
\text { (occurrence) }\end{array}$ & $\begin{array}{l}\text { Standard } \\
\text { dispersion }\end{array}$ & No dispersion & $\begin{array}{l}\text { Anomalous } \\
\text { dispersion }\end{array}$ \\
\hline All data (558) & $0.41^{\circ}(177)$ & $0.05^{\circ}(351)$ & $-0.43^{\circ}(30)$ \\
Without LLPP (328) & $0.38^{\circ}$ & $0.06^{\circ}$ & $0.38^{\circ}$ \\
With LLPP (230) & $0.43^{\circ}$ & $0.04^{\circ}$ & $0.30^{\circ}$ \\
\hline
\end{tabular}

We also studied the activity (AE index) dependence of different characteristics. Figure 8a shows that the relative occurrence of IB dispersion types is similar for three different levels in the AE index, and Fig. 8b shows the same for relative occurrence of LLPPs. The absence of visible AE dependence (using $1 \mathrm{~h}$ average values) is not quite clear for us; however we have to caution that there were no storms and very few strong activity events in September 2009.

The latitudinal differences between the isotropy boundaries in the 30 and $80 \mathrm{keV}$ energy channels in CSS-like dispersion events are displayed in Fig. 9 (bottom). The values above $0.5^{\circ}$ (comparable to the model predictions using the T96 model) are rather rare; most of points cluster at lower values, so the average latitudinal shift is about $0.4^{\circ}$ CGLat. Another important number corresponds to the latitudinal difference between possible poleward-most (IB0 or IB1) and equatorward-most (IB2) positions of true isotropy boundaries in the numerous class of structured (multiple) IBs. (The notations IB0, IB1 and IB2 are illustrated in Fig. 4 and the corresponding caption.) As follows from Fig. 9 (top), in extreme cases this characteristic of the IB uncertainty can be as large as $2^{\circ}$ CGLat, although its median value varies between $0.5^{\circ}$ and $1^{\circ}$, being slightly larger for the $80 \mathrm{keV}$ channel and showing a tendency to increase from premidnight to postmidnight MLT hours.
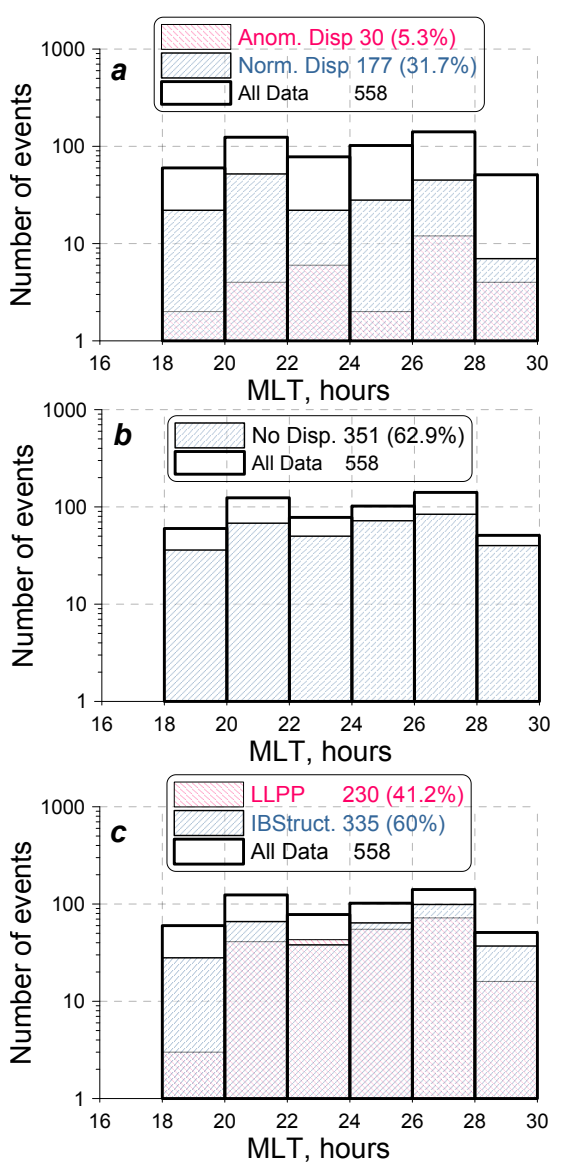

Figure 7. MLT distribution of different IB dispersion types (a) and for the groups showing structured IBs and LLPP (b).

\section{Discussion}

As discussed in the Introduction, the current sheet scattering (CSS) mechanism is commonly accepted as the main mechanism which scatters protons in the plasma sheet (to form their isotropic distributions) and provide the extended regions of their isotropic precipitation in the auroral oval. Inside the tail current sheet where $R_{c} / \rho$ falls well below $K_{\mathrm{cr}}=8$, the particle pitch-angle change during each of the crossings of the current sheet is so strong (some tens of degrees) that this robust scattering mechanism quickly destroys the proton angular anisotropy. However, in the magnetic flux tubes near the isotropy boundary, where $R_{c} / \rho \sim 8$, the pitch-angle change is about the size of the loss cone, as small as just $1-2^{\circ}$. In this case some pitch-angle anisotropy may survive. In contrast with the more distant plasma sheet domain, here and in the inner region the plasma instabilities can grow, which require some proton anisotropy to be excited, like in the case of EMIC waves. Therefore, two competing mechanisms, CSS and wave-induced scattering, can in principle be both at play in the vicinity of the proton isotropy boundary. 

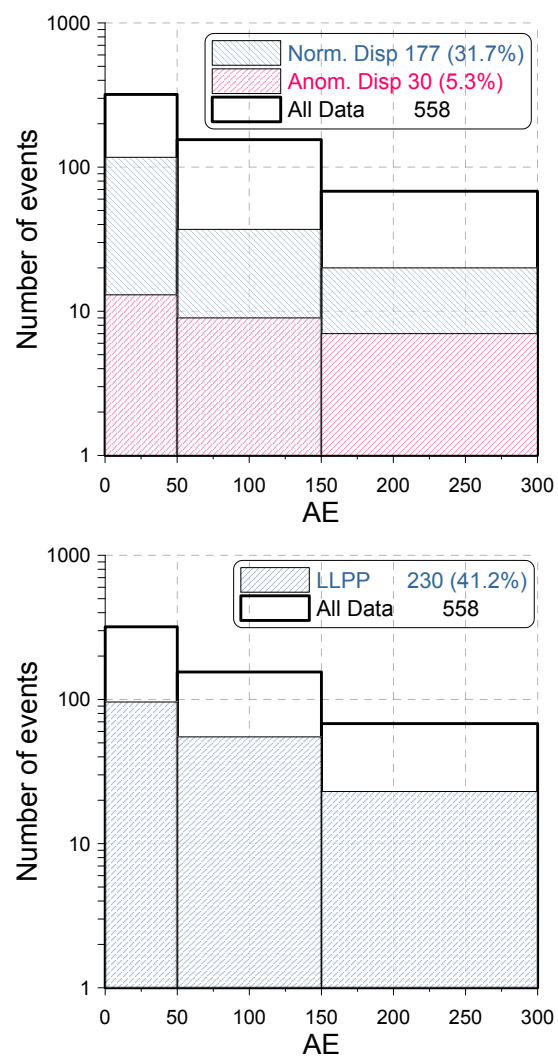

Figure 8. AE activity dependence of different IB dispersion types (a) and of LLPP occurrence.

The distinctive feature of the CSS mechanism is a specific energy-latitude dispersion pattern near the equatorward boundary of proton IB, with a sharp isotropic precipitation boundary occurring at higher (lower) latitude for protons having lower (higher) energy. Such dispersions have been previously demonstrated in the magnetosphere (West et al., 1978), at low altitudes (Imhof, et al. 1977, 1979; Sergeev et al., 2012; Yue et al., 2013) and at middle altitudes (a few $R_{\mathrm{e}}$ ) (e.g., Ganushkina et al., 2005). This forms the basic frame of energy-latitude dispersion, which does not change between quiet and disturbed conditions (see, e.g., Fig. 3). However, the real picture is not always that simple, as has been recently shown by Liang et al. (2014) and in the present paper. As follows from the study presented in Sect. 2.1, the dispersion patterns have details which may vary from one auroral zone crossing to another, although they are truly spatial (but slowly changing) structures as follows from our study of two-satellite observations made in "string-of-pearls" flying configuration (Sect. 2.1).

However, the results for auroral protons $(<20 \mathrm{keV}$; Liang et al., 2014) and for energetic protons $(>30 \mathrm{keV}$, present study) are different. At low proton energies (1-20 keV), the cases with clear reverse energy dispersion pattern are frequent $(77$ reverse dispersion events against 108 standard dispersion events); they often occupy the entire $1-20 \mathrm{keV}$ en-
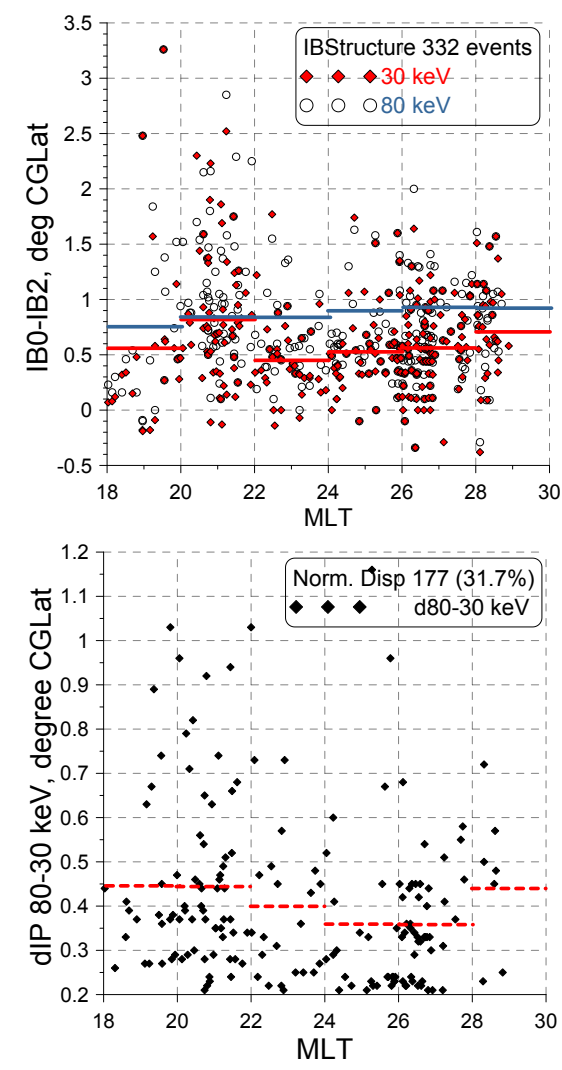

Figure 9. MLT distributions of the latitudinal shift between IBs at 30 and $80 \mathrm{keV}$ for normal dispersion events ( $\mathrm{dIB}$, bottom) and between poleward-most and equatorward-most IBs at $30 \mathrm{keV}$ in the group of multiple structured IBs (IB0-IB2, top).

ergy range and tend to be seen preferentially in the postmidnight MLT sector (Fig. 2 of Liang et al., 2014). However, for energetic protons, the reverse dispersion pattern never occupies the entire energy range; the reverse (anomalous) dispersion between 30 and $>80 \mathrm{keV}$ energies are rare $(5 \%)$ and they show only a slight tendency for the MLT variation (Fig. 7). These differences between low-energy and high-energy proton dispersions are not surprising, taking into account the fact that wave-particle interactions are energyselective process and that only low-energy protons are subject to the field-aligned acceleration, which can also contribute to the apparent deviations in the energy-latitude dispersion pattern. Also, the EMIC waves that may efficiently pitch-angle-scatter the $>30 \mathrm{keV}$ protons are more likely $\mathrm{He}^{+}$ band rather than $\mathrm{H}^{+}$band, so the differences may actually reflect the statistical characteristics of He-band EMIC waves.

Summarizing our findings from studying the latitudinal shifts between the 30 and $80 \mathrm{keV}$ proton IBs, we focus on two basic deviations from the CSS prediction. The first is that the structured IB patterns (multiple IBs) are common, and the second is that the latitude-energy dispersion scale is usually much smaller compared to the expectations from model computations. Even for the standard dispersion event 
group, the average shift $\left(0.4^{\circ}\right)$ is half as large compared to the T96 predictions mentioned in the Sect. 2; this value is actually not far from the low limit $\left(0.2^{\circ}\right)$ allowed for this group. Also, the standard dispersion events are two times less frequent as compared to the events showing no latitude-energy dispersion (Sect. 2.2). Previously, similar conclusions of very steep rigidity-dependent IB latitudinal profile have been obtained in a study of several growth phase events by Sergeev et al. (2012), which covered the entire available rigidity range between $30 \mathrm{keV}$ electrons and $250 \mathrm{keV}$ protons.

In terms of the pure CSS model, the steep IB profile may be explained by very large but localized $\mathrm{d} B_{z} / \mathrm{d} r$ magnetic field gradients (see, e.g., Fig. 10). For example, let us suggest that IBs of 30 and $80 \mathrm{keV}$ protons fall within the latitudinal distance of $0.2^{\circ}$ and that $\mathrm{d} B_{x} / \mathrm{d} z$ is not changing much on this small radial scale in the equatorial plane. From the criterion $R_{c} / \rho \approx B z^{2} /\left(\mathrm{d} B_{r} / \mathrm{d} z \cdot G\right)=8$, one then obtains the scaling $B_{z_{80}} / B_{z_{30}}=(80 / 30)^{1 / 4}=1.28\left(B_{z_{80}}\right.$ and $B_{z_{30}}$ are $B_{z}$ values at the equatorial IB mapping locations for corresponding energies 80 and $30 \mathrm{keV}$ ). Numerically, with radial scale $0.2 R_{\mathrm{e}}$ corresponding to the latitudinal shift $0.2^{\circ}$ in T96 models, this implies a $B_{z}$ change of $9 \mathrm{nT}$ for the mean field of $B_{z_{30}}=30 \mathrm{nT}$. For reference, the gyroradius of $36 \mathrm{keV}$ protons in the $30 \mathrm{nT}$ magnetic field is $0.14 R_{\mathrm{e}}$. Such a locally enhanced gradient comparable to a few proton gyroradius scales is not impossible. For example, in the region of interest (6-8 $R_{\mathrm{e}}$ ), a similar radially localized "magnetic wall" can be formed by the enhanced convection during the substorm growth phase, as has been shown numerically by particle tracing in the self-consistent magnetic field of Rice Convection Model (RCM) simulation (see Figs. 4 and 5 of Yang et al., 2013). Narrow structures of plasma pressure and currents can also form during the flow burst interaction with the plasma sheet (Yang et al., 2014). Previously, thin plasma boundaries (of $30 \mathrm{keV}$ proton gyroradius scale) have been inferred based on Cluster observations and interpreted as a result of flow burst interaction with the inner magnetosphere (Sergeev et al., 2003). In addition, one may also speculate that the scattering threshold condition $R_{c} / \rho \approx B_{z}^{2} /\left(\mathrm{d} B_{r} / \mathrm{d} z \cdot G\right)=8$ should also be modified in the presence of sharp (energetic proton gyroscale) radial $B$-field structures, and this may be an interesting topic for a future study.

However, it is hard to form the magnetic wall on a much larger radial scale and for extended time periods, as occurs frequently in our case. The multiplicity of "nearby IB structures" can in principle be produced by the fluctuating $B_{z}$ radial profile (e.g., formed by the multiple flow bursts). However, it is unlikely that multiple IB structures of that kind, which span $1-2^{\circ}$ CGLat, may continue and exist for half of all observation time, especially during quiet periods. The appearance of "magnetic-wall-type" gradients should be tested observationally in future, for example by using inbound or outbound equatorial traversals of multiple THEMIS spacecraft across the inner magnetosphere.

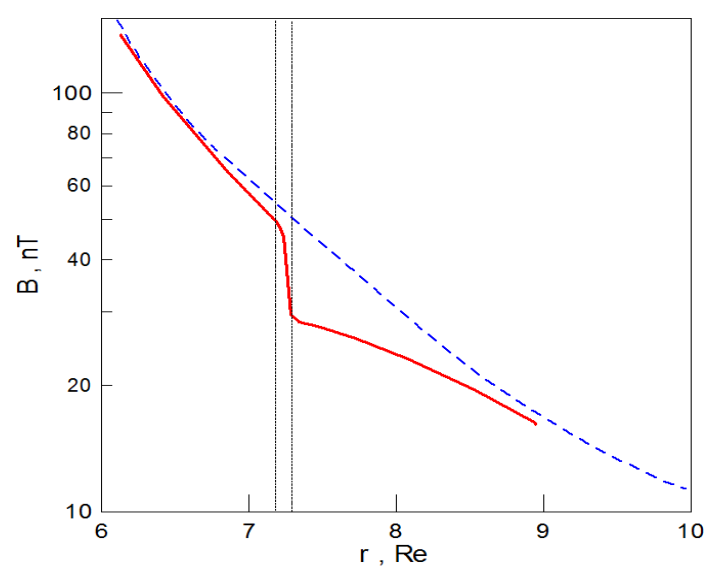

Figure 10. Undisturbed equatorial $B_{z}$ profile (blue dashed line) and hypothetical "magnetic-wall-type" profile (red line) which can provide "no-dispersion" isotropy boundary signature.

Another possibility to explain unusually sharp (and multiple) variations in the $J_{0} / J_{90}$ ratio is to suggest that the resonant wave-particle interaction acts in the plasma tubes near the proton isotropy boundaries, e.g., when the EMIC waves are excited there, as shown by Liang et al. (2014). The perpendicular pressure anisotropy, which is required to excite the EMIC waves (e.g., Cornwall et al., 1970), can be naturally produced by the inward convection acting in the strong $B$-gradient region (e.g., Smets et al., 1999) where the proton IB is located. As already mentioned above, the pitchangle scattering amplitudes near the IB are small (about $1-2^{\circ}$ during one current sheet traversal), so they may not destroy the buildup of pressure anisotropy due to the (large-scale or localized) inward convection. In the presence of proton anisotropy, the sharp (and multiple) variations in the $J_{0} / J_{90}$ ratio can be mediated by the spatial variations in some other parameter, by either the plasma density or magnetic field. However, all these aspects should be combined and tested in a quantitative model.

Common appearance of apparent multiple IBs puts constraints on using the proton IBs to monitor the magnetotail configuration and its changes. First, the latitudinal width of $1^{\circ}$ CGLat of the region of multiple IBs $\left(2^{\circ}\right.$ in the extreme cases) characterizes an uncertainty in the current IB determinations. If the wave-induced processes are responsible for this phenomenon, the usage of the poleward-most boundary of registered multiple IBs can be recommended. Second, in this case the usage of equatorward-most IB (IB2 in the case of Fig. 5a) together with the criterion $R_{c} / \rho \approx 8$ would result in a systematic error of $1-2^{\circ}$ compared to the actual results. Particularly, this can partly account for a $1-2^{\circ}$ disagreement between predicted and observed isotropy boundaries in the testing of adapted models by Shevchenko et al. (2010). Further investigations of the proton-scattering mechanism, acting in the vicinity of proton isotropy boundaries, are highly desirable. These should include the observational THEMIS- 
or CLUSTER-based studies of waves and magnetic field structures in the equatorial magnetosphere region which is conjugate to the IBs, as well as the trajectory simulations of magnetic moment conservation and angular particle scattering in the presence of possible large radial $B_{z}$ gradients.

\section{Conclusions}

Whereas, on a global scale, the rigidity-dependent pattern of isotropy boundaries of protons and electrons is organized according to the current sheet scattering (CSS) mechanism, our study has shown that the dispersion pattern of $30-100 \mathrm{keV}$ energy proton IBs represents a variable spatial structure, which often shows unexpectedly low latitude-energy dispersion (between 30 and $80 \mathrm{keV}$ energies of protons) and multiple IB-like transitions of (precipitated to trapped) flux ratio, in addition to less frequent CSS-type standard dispersion. The region of structured flux ratio has the scale size of about $1^{\circ}$, which limits the accuracy of proton IB determination to be used for magnetotail monitoring and applications. This combination of facts (most probably) points out that another mechanism, the wave-induced particle scattering (probably by EMIC waves), acts effectively in the plasma tubes extending $\sim 1 R_{\mathrm{e}}$ earthward from the nominal boundary between the adiabatic and non-adiabatic particle motion boundary.

Acknowledgements. This study was supported by the Russian Foundation of Basic Research (grant 13-05-00132). The NOAAPOES particle data were provided by the NOAA National Geophysical Data Center (http://ngdc.noaa.gov). The work by N. Y. Ganushkina and S. V. Dubyagin was partly supported by the Academy of Finland. The work by N. Y. Ganushkina was also partly supported through NASA award NNX14AF34G.

The topical editor E. Roussos thanks J. Liang and one anonymous referee for help in evaluating this paper.

\section{References}

Alfven, H. and Falthammar, C. G.: CosmicalElectrodynamics, Fundamental Principles, 2nd ed., Clarendon, Oxford, 1-260, 1963.

Asikainen, T., Mursula K., and Maliniemi, V.: Correction of detector noise and recalibration of NOAA/MEPED energetic proton fluxes, J. Geophys. Res., 117, A09204, doi:10.1029/2012JA017593, 2012.

Cornwall, J. M., Coroniti, F. V., and Thorne, R. M.: Turbulent loss of ring current protons, J. Geophys. Res., 75, 4699-4709, 1970.

Delcourt, D. C., Sauvaud, J.-A., Martin Jr., R. F., and Moore, T. E.: On the nonadiabatic precipitation of ions from the near-Earth plasmasheet, J. Geophys. Res., 101, 17409-17418, 1996.

Donovan, E. F., Jackel, B. J., Voronkov, I., Sotirelis, T., Creutzberg, F., and Nicholson, N. A.: Ground-based optical determination of the b2iboundary: A basis for an optical MT-index, J. Geophys. Res., 108, 1115, doi:10.1029/2001JA009198, 2003.

Dubyagin, S., Ganushkina, N., Apatenkov, S., Kubyshkina, M., Singer, H., and Liemohn, M.: Geometry of duskside equatorial current during magnetic storm main phase as deduced from magnetospheric and low-altitude observations, Ann. Geophys., 31, 395-408, doi:10.5194/angeo-31-395-2013, 2013.

Evans, D. S. and Greer, M. S.: Polar orbiting environmental satellite space environment monitor-2: Instrument descriptions andarchive data documentation, NOAA Tech. Memo. 1.4, Space Environ. Lab., Boulder, Colo, 2004.

Ganushkina, N. Yu., Pulkkinen, T. I., Kubyshkina, M. V., Sergeev, V. A., Lvova, E. A., Yahnina, T. A., Yahnin, A. G., and Fritz, T.: Proton isotropy boundaries as measured on mid- and low-altitude satellites, Ann. Geophys., 23, 1839-1847, doi:10.5194/angeo23-1839-2005, 2005.

Gvozdevsky, B. B., Sergeev, V. A., and Mursula, K.: Long lasting energetic proton precipitation in the inner magnetosphere after substorms, J. Geophys. Res., 102, 24333-24338, 1997.

Horne, R. B., Thorne, R. M., Shprits, Y. Y., Meredith, N. P., Glauert, S. A., Smith, A. J., Kanekal, Sh. G., Baker, D. N., Engebretson, M. J., Posch, J. L., Spasojevic, M., Inan, U. S., Pickett, J. S., and Decreau, P. M. E.: Wave acceleration of electrons in the Van Allen radiation belts, Nature, 437, 227-230, doi:10.1038/nature03939, 2005.

Imhof, W. L., Reagan, J. B., and Gaines, E. E.: Fine scale spatial structure in the pitch angle distributions of energetic particles near the midnight trapping boundary, J. Geophys. Res., 82, 5215, 1977.

Imhof, W. L., Reagan, J. B., and Gaines, E. E.: Studies of the sharplydefined L dependent energy threshold for isotropy at the midnight trapping boundary, J. Geophys. Res., 84, 6371-6384, 1979.

Liang, J., Donovan, E., Ni, B., Yue, C., Jiang, F., and Angelopoulos, V.: On an energy-latitude dispersion pattern of ion precipitation potentially associated with magnetospheric EMIC waves, J. Geophys. Res. Space Physics, 119, 8137-8160, doi:10.1002/2014JA020226, 2014.

Meurant, M., Gérard, J.-C., Blockx, C., Spanswick, E., Donovan, E. F., Hubert, B., Coumans, V., and Connors, M.: EL - a possible indicator to monitor the magnetic field stretching at global scale during substorm expansive phase: Statistical study, J. Geophys. Res., 112, A05222, doi:10.1029/2006JA012126, 2007.

Newell, P. T., Sergeev, V. A., Bikkuzina, G. R., and Wing, S.: Characterizing the state of the magnetosphere: Testing the ion precipitationmaxima latitude (b2i) and the ion isotropy boundary, J. Geophys. Res., 103, 4739-4745, doi:10.1029/97JA03622, 1998.

Nishimura, Y., Bortnik, J., Li, W., Thorne, R. M., Lyons, L. R., Angelopoulos, V., Mende, S. B., Bonnell, J., Le Contel, O., Cully, C., Ergun, R., and Auster, U.: Estimation of magnetic field mapping accuracy using the pulsating aurora-chorus connection, Geophys. Res. Lett., 38, L14110, doi:10.1029/2011GL048281, 2011.

Sergeev, V. A. and Gvozdevsky, B. B.: MT-index - a possible new index to characterize the magnetic configuration of magnetotail, Ann. Geophys., 13, 1093-1103, doi:10.1007/s00585-995-10939, 1995.

Sergeev, V. A. and Tsyganenko, N. A.: Energetic particle losses and trapping boundaries as deduced from calculations with a realisticmagnetic field model, Planet. Space Sci., 10, 999-1006, 1982.

Sergeev, V. A., Sazhina, E. M., Tsyganenko, N. A., Lunbland, J. A., and Soraas, F.: Pitch angle scattering of energetic pro- 
tons in themagnetotail current sheet as the dominant source of their isotropic precipitation into the nightside ionosphere, Planet. Space Sci., 31, 1147-1158, 1983.

Sergeev, V. A., Malkov, M., and Mursula, K.: Testing the isotropic boundary algorithm method to evaluate the magnetic field configuration in the tail, J. Geophys. Res., 98, 7609-7620, doi:10.1029/92JA02587, 1993.

Sergeev, V. A., Sauvaud, J.-A., Reme, H., Balogh, A., Daly, P., Zong, Q.-G., Angelopoulos, V., Andre, M., and Vaivads, A.: Sharp boundary between the inner magnetosphere and active outer plasma sheet, Geophys. Res. Lett., 30, 1799, doi:10.1029/2003GL017095, 2003.

Sergeev, V., Nishimura, Y., Kubyshkina, M., Angelopoulos, V., Nakamura, R., and Singer, H.: Magnetospheric location of the equatorward prebreakup arc, J. Geophys. Res., 117, A01212, doi:10.1029/2011JA017154, 2012.

Shevchenko, I. G., Sergeev, V., Kubyshkina, M., Angelopoulos, V., Glassmeier, K. H., and Singer, H. J.: Estimation of magnetosphere-ionosphere mapping accuracy using isotropy boundary and THEMIS observations, J. Geophys. Res., 115, A11206, doi:10.1029/2010JA015354, 2010.

Smets, R., Delcourt, D., Sauvaud, J. A., and Koperski, P.: Electron pitch angle distributions following thedipolarization phase of a substorm: Interball-Tail observations and modeling, J. Geophys. Res., 104, A7, 14571-14581, 1999.

Usanova, M. E., Mann, I. R., Kale, Z. C., Rae, I. J., Sydora, R. D., Sandanger, M., Søraas, F., Glassmeier, K.-H., Fornacon, K.-H., Matsui, H., Puhl-Quinn, P. A., Masson, A., and Valliéres, X.: Conjugate ground and multisatellite observations of compression-related EMIC Pc1 waves and associatedproton precipitation, J. Geophys. Res., 115, A07208, doi:10.1029/2009JA014935, 2010.
Wang, C.-P., Zaharia, S. G., Lyons, L. R., and Angelopoulos, V.: Spatial distributions of ion pitchangle anisotropy in the near-Earth magnetosphere and tail plasma sheet, J. Geophys. Res. Space Physics, 118, 244-255, doi:10.1029/2012JA018275, 2012.

West Jr. , H., Buck, R., and Kivelson, M.: On the Configuration of themagnetotail near midnight during quiet and weakly disturbed periods:Magnetic field modeling, J. Geophys. Res., 83, 38193829, 1978.

Yahnin, A. G. and Yahnina, T. A.: Energetic proton precipitation related to ion-cyclotron waves, J. Atmos. Terr. Phys., 69, 16901706, 2007.

Yang, J., Wolf, R. A., Toffoletto, F. R., and Sazykin, S.: RCM-E simulation of substorm growth phase arc associated with largescale adiabatic convection, Geophys. Res. Lett., 40, 6017-6022, doi:10.1002/2013GL058253, 2013.

Yang, J., Toffoletto, F. R., and Wolf, R. A.: RCM-E simulation of a thin arc preceded by a north-south-aligned auroral streamer, Geophys. Res. Lett., 41, 2695-2701, doi:10.1002/2014GL059840, 2014.

Yue, C., Wang, C.-P., Zaharia, S. G., Xing, X., and Lyons, L.: Empirical modeling of plasma sheet pressure and three-dimensional force-balanced magnetospheric magnetic field structure: 2 . Modeling, J. Geophys. Res. Space Physics, 118, 6166-6175, doi:10.1002/2013JA018943, 2013.

Yue, C., Wang, C.-P., Donovan, E. F., Zaharia, S. G., Lyons, L., Liang, J., and Henderson, M.: Current sheet scattering and ion isotropicboundary under 3D empirical force-balanced magnetic field, J. Geophys. Res. Space Physics, 119, 8202-8211, doi:10.1002/2014JA020172, 2014. 